\title{
Validación del Cuestionario de Conciencia Emocional en adolescentes ESPAÑOLES*
}

\section{Validation of Emotional Awareness Questionnaire in Spanish adolescents}

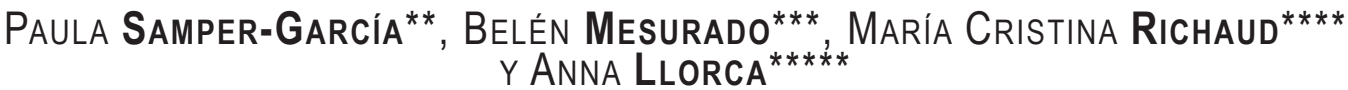

\begin{abstract}
*Trabajo realizado en el marco de una Beca Postdoctoral otorgada a la Dra. Belén Mesurado por la Fundación Carolina (España) y la Universidad Austral (Argentina) Ref. 478341 - 2013-2014.

**Doctora en Psicología. Profesora Titular de Psicología Básica en el Departamento de Psicología Básica de la

Universidad de Valencia. E-Mail: paula.samper@uv.es

Avda. Blasco Ibáñez, 13. 46010 Valencia. España.

${ }^{* * *}$ Doctora en Psicología. Miembro de la Carrera del Investigador Científico del Consejo Nacional de Investigaciones

Científicas y Técnicas (CONICET).

${ }^{* * * *}$ Doctora en Psicología. Miembro de la Carrera del Investigador Científico del Consejo Nacional de Investigaciones Científicas y Técnicas (CONICET).

*****MA en Psicología. Becaria del Departamento de Personalidad, Evaluación y Tratamientos Psicológicos de la Universidad de Valencia.
\end{abstract}

\section{REsUMEN}

La regulación de la emoción es un proceso complejo que implica la iniciación, inhibición o modulación del propio estado o comportamiento en una situación dada (Cole, Michel \& Teti, 1994). La regulación emocional tiene una función altamente significativa en la vida humana ya que reacciones emocionales inapropiadas, extremas o incontroladas pueden impedir un ajuste funcional a la sociedad (Koole, 2009). Sin embargo para una adecuada regulación emocional es necesaria una buena comprensión emocional y, a su vez, para una comprensión eficaz se requiere una apropiada percepción emocional. En este estudio se analizan las propiedades psicométricas de la versión española del Cuestionario de Conciencia Emocional revisado (Emotional Awareness Questionnaire, EAQ30) desarrollado por Rieffe, Oosterveld, Miers, Terwogt y Ly (2008). La versión española del EAQ30 fue administrada a 1.316 adolescentes españoles de 14 a 16 años. Los datos confirman la estructura original de la escala de seis factores: Distinción de las emociones, Intercambio verbal de las emociones, No ocultamiento de las emociones, Conciencia corporal, Análisis de las emociones y Atención a las emociones de los otros. Los coeficientes de consistencia interna de las subescalas del EAQ30 fueron satisfactorios y equivalentes a los obtenidos en la escala original. Por otra parte, el estudio también confirmó la invariancia de la estructura factorial del instrumento cuando se considera el sexo, en la adolescencia media. Estos resultados permitirían sostener que la adaptación al español aquí presentada puede ser utilizada para evaluar el constructo conciencia emocional en adolescentes de lengua española de un modo válido y confiable en ambos sexos.

Palabras clave: Conciencia emocional; Validación; Fiabilidad; Adolescentes. 
ABSTRACT

Emotional awareness is an attentional process that enables us to monitor our emotions and to differentiate between various emotions in a qualitative sense, to locate their antecedents, and to acknowledge the physiological correlates of the emotion experience (Rieffe, Oosterveld, Miers, Terwogt, \& Ly, 2008).

Rieffe and colaborators (2008) consider that emotional awareness also includes attitudinal aspects such as positive or negative appreciation of the own emotions, consideration of them as aspects of oneself, or on the contrary, that they must be communicated to the others. Emotional awareness would be a cognitive skill that would enable the opportunity to regulate the most primitive emotional reactions and find patterns that are more appropriate reaction to a particular context (Rieffe, Terwogt, Petrides, Cowan, Miers, \& Tolland, 2007). It is necessary to have a good emotional awareness to adequately cope a particular situation.

Since there were no scales to measure emotional awareness but often alexithymia scales were used to evaluate it as its opposed emotional state, Rieffe and colleagues (2007) developed the Emotional Awareness Questionnaire (EAQ).

Different analyses carried out by Rieffe and collaborators led to the Revised Emotional Awareness Questionnaire (EAQ Revised - Rieffe et al., 2008). Subsequent studies developed by Lahaye, Luminet, Broeck, Bodart, and Mikolajczak (2010) using the revised Emotional Awareness Questionnaire, found a positive correlation of the questionnaire's six dimensions with emotional intelligence. The same study showed a negative correlation between emotional awareness and the three dimensions difficulty in identifying feelings, difficulty in describing feelings, and oriented to an external thinking - that make up Alexithymia Questionnaire for Children developed by Rieffe, Oosterveld, and Meerum Terwogt (2006).

Given the importance of emotional awareness to the emotional development of children and adolescents and that there are no instruments published in Spanish that validly measure this construct, in this study, we investigated the psy- chometric properties of the Spanish version of the Emotional Awareness Questionnaire (EAQ30; Rieffe et al., 2008). The EAQ30 is a self-report questionnaire comprising 30 items rated on a 3 point scale $(1=$ Not true, $2=$ Sometimes true, $3=$ True). It includes six subscales or dimensions: (1) Differentiating emotions or ability to differentiate and understand the causes of emotions; (2) Bodily awareness or identification of the physical aspects of emotional experience; (3) Verbal sharing or verbal communication's own emotional state; (4) Acting out emotions or impulsive tendency to show emotions of oneself in a way non- functional; (5) Analyses of emotions or ability to deal voluntarily to one's emotions; and finally, and (6) Attending to others' emotions or the voluntary decision to deal with the emotions of others. The EAQ30 was administered to 1316 children aged 14 to 16 years old (age: $M=14.95 ; S D=.72$ ), from Valencia (Spain), of middle socioeconomic level. The translation of this questionnaire was performed according to the International Test Commission guidelines for test adaptation (Hambleton, 2001). A six-factor model was tested using AMOS Program; the results have shown that the original 6-factor structure was replicated in our data.

The internal consistency coefficients of the EAQ30 subscales were satisfactory -Cronbach's estimates between .68 and .70- and equivalent to those obtained in the original scale. A multiple group analysis was used to test whether the sixfactor model was invariant across the gender by examining the change in model ji square and comparative fit index (CFI) values. We found the model of 6-factor has metric invariant through gender. These results allow us to claim that the Spanish version presented here can be used to evaluate the construct emotional awareness in Spanish adolescents of a valid and reliable way.

It would be interesting to do further studies with different Spanish-speaking populations to see if the stability of the structure is preserved after making appropriate adjustments to the specific language in the use of Spanish for each of the populations involved.

Key words: Emotional awareness; Validity; Reliability; Adolescents. 


\section{INTRODUCCIÓN}

Las emociones desempeñan un importante rol en el bienestar y el malestar psicológico, así como en la adaptación y la regulación de las relaciones interpersonales (Schutte, Malouff, Thorsteinsson, Bhullar \& Rooke, 2007). A diario las personas están expuestas a una gran variedad de estímulos potencialmente activantes. Por ello es muy importante su capacidad para regular la emoción. La regulación de la emoción es un proceso complejo que implica la iniciación, inhibición o modulación del propio estado o comportamiento en una situación dada (Cole, Michel \& Teti, 1994). La regulación emocional tiene una función altamente significativa en la vida humana ya que reacciones emocionales inapropiadas, extremas o incontroladas pueden impedir un ajuste funcional a la sociedad (Koole, 2009). Sin embargo, para una adecuada regulación emocional es necesaria una buena comprensión emocional y, a su vez, para una comprensión eficaz se requiere una apropiada percepción emocional.

En los últimos años ha surgido un nuevo concepto denominado conciencia emocional que ha sido objeto de investigación como factor protector de la salud física y psíquica en la niñez. La conciencia emocional es entendida como un proceso atencional que implica funciones interpretativas y evaluativas y permite monitorear las propias emociones, diferenciarlas unas de otras, identificar sus causas y conocer las correlaciones fisiológicas de la experiencia emocional (Rieffe, Oosterveld, Miers, Terwogt \& Ly, 2008).

Rieffe y colaboradores (2008) consideran que la conciencia emocional también incluye aspectos de tipo actitudinal, tales como la valoración positiva o negativa de las propias emociones, la consideración de que son aspectos privados de uno mismo, o que por el contrario, deben ser comunicados a los demás. La conciencia emocional sería una habilidad cognitiva que posibilitaría la oportunidad de regular las reacciones emocionales más primitivas y encontrar patrones de reacción que sean más apropiados a un contexto determinado (Rieffe, Terwogt, Petrides, Co- wan, Miers \& Tolland, 2007). Es necesario tener una buena conciencia emocional para afrontar adecuadamente una situación particular.

Existe evidencia de que la incapacidad para identificar las propias emociones y describirlas verbalmente -alexitimia (Sifneos, 1973)- o dicho de otra manera, el déficit en la conciencia emocional, está directamente asociada con la vulnerabilidad psicológica, el estado anímico depresivo y la hostilidad, como manifestación abierta de la ira (Ruedas, Pérez Gracía, San Juan \& Ruiz, 2006).

Dado que no existían escalas para medir la conciencia emocional, sino que muchas veces se utilizaban las escalas de alexitimia para evaluarla como su estado emocional contrapuesto, Rieffe y colaboradores (2007) desarrollaron el Cuestionario de Conciencia Emocional (Emotional Awareness Questionnaire, EAQ). Dicho cuestionario es un autoinforme para niños, compuesto por seis componentes: (1) la Distinción de las emociones o habilidad para diferenciar y comprender las causas de las propias emociones, (2) el Intercambio verbal de las emociones o la comunicación verbal del propio estado emocional, (3) la Reacción emocional o la tendencia impulsiva a mostrar las emociones de uno mismo de un modo no funcional, por ejemplo, "cuando estoy enojado, me gusta que todos lo sepan", (4) la Conciencia corporal o la identificación de los aspectos físicos de la experiencia emocional, (5) el Análisis de las emociones o la capacidad de enfrentarse voluntariamente a las propias emociones y (6) la Atención a las emociones de los demás o la decisión voluntaria de enfrentarse a las emociones de las otras personas (Rieffe et al., 2007).

Posteriores análisis llevados a cabo por Rieffe y colaboradores (2007) con el objetivo de analizar la validez predictiva del Cuestionario Multidimensional de Conciencia Emocional, mostraron que las subescalas Distinción de las emociones, Intercambio verbal de las emociones y Análisis de la emociones estaban inversamente relacionadas con la depresión en los niños; las dimensiones Distinción de las emociones e Intercambio ver- 
bal de las emociones tenían una relación negativa con los síntomas de preocupación; Atención a las emociones de los demás y Conciencia corporal estaban directamente asociados con los síntomas de preocupación y finalmente, Distinción de las emociones y Análisis de la emociones tenían una relación inversa con las quejas somáticas mientras que la Conciencia corporal y Atención a las emociones de los demás tenían una relación positiva. En síntesis, todas las dimensiones que componen la escala se encontraban asociadas a diferentes problemas psicológicos, a excepción de la dimensión Reacción emocional.

Rieffe y colaboradores retomaron los estudios del análisis del Cuestionario de Conciencia Emocional en el año 2008, e hicieron notar que los ítems que componían la dimensión Reacción emocional en su escala original, representaban dos aspectos opuestos, por un lado la tendencia a expresar las emociones y por el otro, la tendencia a ocultarlos. Es probable que este fuera el motivo por el cual esta dimensión no predecía los problemas psicológicos evaluados en los niños. En una revisión del Cuestionario de Conciencia Emocional (EAQ Revisado) realizada por los autores, se eliminaron los ítems que hacían referencia a la tendencia a ocultar las emociones y se re-denominó la dimensión No ocultamiento de las emociones. Este nueva dimensión mostró fuertes correlaciones con todos los índices de internalización de problemas y especialmente con la ansiedad social y la preocupación (Rieffe et al, 2008).

Posteriores estudios desarrollados por Lahaye, Luminet, Broeck, Bodart y Mikolajczak (2010) utilizando la versión revisada del Cuestionario de Conciencia Emocional, encontraron una correlación positiva de las seis dimensiones del cuestionario con la inteligencia emocional. El mismo estudio mostró una correlación negativa entre la conciencia emocional y las tres dimensiones -dificultad para identificar los sentimientos, dificultad para describir los sentimientos y orientado hacia un pensamiento externo- que componen el Cuestionario de Alexitimia para Niños desa- rrollado por Rieffe, Oosterveld y Meerum Terwogt (2006).

Dada la importancia que tiene la conciencia emocional para el desarrollo emocional de los niños y adolescentes y que no existen instrumentos publicados en español que midan válidamente este constructo, el objetivo del trabajo que se informa fue validar la estructura factorial del modelo de conciencia emocional planteado por Rieffe y colaboradores y de la fiabilidad medida como consistencia interna a través del Alpha de Cronbach, de la versión en español de la EAQ30 revisada, en una muestra de adolescentes españoles. Además se analizó la invariancia del modelo de seis factores en función del sexo.

\section{MÉTOdO}

\section{PARTICIPANTES}

El muestreo fue de tipo no probabilístico y participaron del estudio 1.316 adolescentes españoles de entre 14 y 16 años de edad $(M=14.95 ; D T=.72)$ de ambos sexos, 687 varones, con una media de edad igual a 14.99 $(D T=.72)$ y 629 mujeres con una media de edad igual a $14.89(D T=.71)$ de nivel socioeconómico medio, que viven en Valencia (España) y que asisten a escuelas públicas. No se hallaron datos perdidos.

\section{INSTRUMENTO}

La conciencia emocional fue evaluada con el Cuestionario de Conciencia Emocional revisado de Rieffe y colaboradores (2008), que es un autoinforme de 30 ítems con opciones de respuesta que van de 1 a 3 puntos (1: Falso, 2: A veces es verdad y 3: Verdad). El cuestionario permite evaluar 6 dimensiones: Distinción de las emociones (por ejemplo, Es difícil saber si me siento triste, enfadada / o o algo más, ítem inverso); Intercambio verbal de las emociones (por ejemplo, Encuentro difícil explicarle a un/a amigo/a cómo me siento, ítem inverso); No ocultamiento de las emociones (por ejemplo, Me cuesta hablar 
con alguien de cómo me siento, ítem inverso); Conciencia corporal (por ejemplo, Cuando estoy asustada/o o nerviosa/o, siento algo en la barriga); Análisis de las emociones (por ejemplo, Cuando estoy enfadada/o o disgustada/o, intento entender por qué) y Atención a las emociones de los demás (por ejemplo, Es importante saber cómo se sienten mis amiga/os) (ver Protocolo en el Anexo 1).

\section{TRADUCCIÓN DE LOS ÍTEMS}

Para realizar la traducción de los ítems se siguieron los lineamientos propuestos por International Test Commission (Hambleton, 2001). Inicialmente fueron traducidos por una psicóloga que estaba familiarizada con la terminología de la variable evaluada por el instrumento. La traductora conocía la lengua inglesa pero su lengua materna era el español. Se pidió que enfatizara en la traducción conceptual de los ítems y no en hacer una traducción literal, así como que utilizara un lenguaje natural y aceptable para los estudiantes. En un segundo momento, un psicólogo, que también era un experto con experiencia en desarrollo de instrumentos y traducción, revisó la primera traducción para analizar la equivalencia semántica y sintáctica de la versión. Finalmente, el instrumento fue nuevamente traducido a la lengua inglesa por un traductor independiente, cuya lengua materna era el inglés y que no tenía conocimiento del cuestionario original. Al igual que en la traducción inicial, el énfasis se puso en la equivalencia conceptual y cultural y no en la lingüística. Las diferencias fueron revisadas hasta que se alcanzó una versión satisfactoria.

\section{Procedimiento}

La aplicación de los instrumentos se realizó de forma colectiva y en horas de clase, en cada aula y para cada uno de los niveles de edad y cursos seleccionados. Las instrucciones para contestar los cuestionarios se explicaron oralmente. La administración de las pruebas se realizó en una sesión de 15 minu- tos. Previamente a la aplicación de los instrumentos de evaluación se entrevistó a los directores de los centros escolares seleccionados para participar en la investigación, a los profesores y a las familias, con el objetivo de pedir su colaboración, obtener el consentimiento informado y garantizar el anonimato de los datos. Se incluyeron en el estudio todos los alumnos que presentaron el consentimiento informado firmado por sus padres o tutores.

\section{AnÁlisis ESTAdÍsticos}

\section{AnÁlisis Factorial Confirmatorio}

Se realizó un Análisis Factorial Confirmatorio (AFC) para poner a prueba la estructura de seis factores del Cuestionario de Conciencia Emocional con la muestra total. Se estimó el grado de ajuste del modelo teórico a los datos de la muestra a través de la aplicación del Programa AMOS Graphics 7.0 (Arbuckle, 2006). Se evaluó el nivel de bondad de los modelos hipotetizados utilizando la prueba ji cuadrado, los índices de ajuste: GFI (Goodness of Fit Index), AGFI (Adjusted Goodness of Fit Index) y el Indice de Ajuste Comparativo (Comparative Fit Index) CFI. Finalmente, se calculó el RMR (Root Mean Square Residual) y el RMSEA (Root Mean Standard Error). Estos últimos índices son una medida del grado del error del modelo.

\section{ANÁLISIS MULTIGRUPO}

La segunda parte de este estudio fue diseñada para examinar si el mismo modelo factorial y los valores de los parámetros se mantenían a través del sexo. Los análisis se llevaron a cabo a través del procedimiento de análisis multigrupo disponible en el Programa AMOS Graphics 7.0.

Se estudió la consistencia interna del instrumento por medio del cálculo del coeficiente Alpha de Cronbach y la equivalencia de fiabilidad entre la escala original y la adaptación al español. 


\section{Resultados}

La Tabla 1 contiene los puntajes medios y las desviaciones estándares de las dimensiones que componen el Cuestionario de Conciencia Emocional para la muestra total y para cada grupo según el sexo.

\section{AnÁlisis FACTORIAL CONFIRMATORIO}

Antes de realizar el análisis, se decidió estudiar las características de asimetría y curtosis de los datos del modelo de medición para comprobar su normalidad, ya que es un requisito indispensable para realizar una adecuada estimación. Al realizar la prueba se obtuvieron resultados aceptables, pues todos los parámetros se encontraban entre - 1 y 1 . Se realizó un AFC para poner a prueba la estructura factorial de 6 dimensiones del Cuestionario de Conciencia Emocional, utilizando el método de estimación de máxima verosimilitud, dado que los datos del modelo de medición seguían una distribución normal. Los resultados indicaron un buen ajuste entre el modelo propuesto y los datos empíricos. Se obtuvo un $\chi^{2}(390)=1304.84, p \leq .000, \chi^{2} / g l=3.34$. Como se sabe, la hipótesis nula en los modelos de ecuaciones estructurales postula que no hay diferencias entre el modelo teórico y el empírico. Por lo tanto, contrario a la mayoría de las situaciones de puesta a prueba de hipótesis, en el modelo de ecuaciones estructurales es deseable no rechazar la hipótesis nula porque el modelo especificado representa nuestras hipótesis teóricas acerca de la estructura de los datos. Sin embargo como también se sabe que el ji cuadrado es muy sensible al tamaño de la muestra, los autores sugieren tener en cuenta otros índices tales como el $\chi^{2} / g l$, GFI, AGFI, CFI, RMR y RMSEA (Kline, 1998; Milfont \& Fischer, 2010).

En este análisis se obtuvieron los siguientes índices de ajuste: $\mathrm{GFI}=.94 ; \mathrm{AGFI}=.92$, $\mathrm{CFI}=.88$, mostrando todos ellos un ajuste aceptable. Por último, se calculó el RMR (Root Mean Square Residual) y el RMSEA (Root Mean Standard Error) obteniendo
$\mathrm{RMR}=.02$ y RMSEA $=.04$. Como se sabe, valores por debajo de .10 indican un error aceptable y valores alrededor de .06 indican un muy buen ajuste (Hu \& Bentler, 1999).

\section{ANÁLISIS MULTIGRUPO A TRAVÉS DEL SEXO}

Se realizó este análisis con la intención de determinar si el modelo de seis factores se aplicaría igualmente bien a varones y mujeres. Se analizó y se comparó un modelo anidado para examinar los cambios de los valores de ji cuadrado, así como en los demás índices de ajuste del modelo. Los resultados indican que la diferencia entre el ji cuadrado del modelo no restringido y el modelo de peso restringido es no significativo $(p=.06)$; además se analizó la diferencia de los índices de ajuste mostrándose todos ellos invariantes entre ambos grupos (Milfont \& Fischer, 2010). En la Tabla 2 pueden verse los índices que muestran la invariancia del modelo a través del sexo.

En la Tabla 3 se presenta el modelo de ecuaciones estructurales con sus respectivos efectos para cada grupo estudiado.

\section{ESTUDIO DE LA FIABILIDAD}

Para analizar la consistencia interna del Cuestionario de Conciencia Emocional, se ha calculado el coeficiente alpha de Cronbach, obteniéndose un índice de fiabilidad para la escala completa de .74.

\section{ESTUDIO DE LA EQUIVALENCIA EN LA FIABILIDAD}

Para analizar la equivalencia de la fiabilidad entre la escala original y la versión traducida, se estudiaron las diferencias en los alpha de Cronbach. Estas diferencias fueron analizadas con el estadístico $(1-\alpha 1) /(1-\alpha 2)$ que sigue una distribución $F$ con $\left(N_{1}-1\right)$ y $\left(N_{2}-1\right)$ grados de libertad (van der Vijver \& Leung, 1997). Los resultados muestran diferencias estadísticas no significativas entre los valores de alpha obtenidos en la escala ori- 
ginal y los obtenidos con la traducción presentada en este trabajo (ver Tabla 4).

\section{DISCUSIÓN}

Distintos autores han definido los problemas en la regulación de las emociones como dificultades para controlar la influencia de la excitación emocional en la organización y calidad de los pensamientos, acciones e interacciones. Las personas que están emocionalmente desreguladas exhiben patrones de respuesta que tienen un desajuste entre sus objetivos, respuestas y/o modos de expresión y las demandas del ambiente social (Zeman, Cassano, Perry-Parrish \& Stegall, 2006). Mayores niveles de regulación emocional se relacionan probablemente con altos niveles de competencia social y con la expresión socialmente apropiada de las emociones (Fabes et al., 1999). Esta capacidad de regulación de las emociones es sumamente importante para el desarrollo sano de las personas y para su vida en sociedad. A la vez, una de las bases de una buena regulación está en la conciencia emocional, entendida como la capacidad para analizar las emociones, diferenciarlas unas de otras y comprender sus causas. En este sentido, la conciencia emocional tiene un rol central durante la niñez y la adolescencia para establecer buenas relaciones con los demás, así como para favorecer la adaptación al ambiente (Rieffe et al., 2007). Existe creciente evidencia acerca de la asociación entre la conciencia emocional y la inteligencia emocional y la resistencia al estrés, así como también con una mejor salud física y mental (Ferragut \& Fierro, 2009; Jiménez Morales \& López-Zafra, 2007; Mikolajczak, Roy, Luminet, Fillee $\&$ de Timary, 2007; Salovey, Stroud, Woolery $\&$ Epel, 2002). Por otro lado, recientes investigaciones han encontrado que es un buen predictor de la adaptación de los individuos a sus ambientes (Eisenberg, Hofer \& Vaughan, 2007; Thompson \& Meyer, 2007).

Dada la importancia de esta variable y la necesidad de diagnosticar su forma de desarrollo para, en caso de ser necesario, intervenir oportunamente, es crucial contar con ins- trumentos adecuados a las diferentes poblaciones. En el caso del presente trabajo se tuvo por objetivo validar la versión en español del Cuestionario de Conciencia Emocional de Rieffe y colaboradores en su versión revisada y en población española.

Los resultados del estudio muestran que el Cuestionario de Conciencia Emocional en su versión en español y en población española, conserva la estructura factorial de seis dimensiones propuesta por los autores en la versión revisada del original en lengua inglesa. Estos resultados son consistentes con los de estudios previos con la traducción de la escala al francés y en estudios transnacionales realizados por Lahaye y colaboradores (2011).

Por otro lado, se encontraron valores aceptables de consistencia interna en todas las dimensiones que componen la escala. Asimismo, se estudió la equivalencia de los valores alpha de Cronbach de la escala original y los obtenidos en la versión traducida al español, no hallándose diferencias estadísticamente significativas, lo que nos hablaría de la consistencia observada entre la versión original de la escala y la presentada en este estudio.

Por otra parte, el estudio realizado también confirmó la invariancia de la estructura factorial del instrumento cuando se considera el sexo, en la adolescencia media. Estos resultados nos permitirían sostener que la versión en español aquí presentada puede ser utilizada para evaluar el constructo conciencia emocional en adolescentes españoles de un modo válido y confiable.

Sería interesante hacer nuevos estudios con diferentes poblaciones hispano parlantes para ver si se conserva la estabilidad de la estructura una vez realizados los ajustes lingüísticos correspondientes a las particularidades en el uso del español por cada una de las poblaciones implicadas.

El contar con una medida de conciencia emocional con fuertes propiedades psicométricas permitiría entre otros, realizar estudios transculturales y longitudinales que facilten la identificación de los cambios progresivos que pueden llegar a darse en la distintas dimensiones de la conciencia emocional en el transcurso de las diferentes etapas de la adolescencia. 
TABLA 1

ESTAdÍSTICOS DESCRIPTIVOS DE LAS DIMENSIONES QUE COMPONEN EL CUESTIONARIO DE CONCIENCIA EMOCIONAL EN LA MUESTRA TOTAL Y SEGÚN SEXO

\begin{tabular}{|c|c|c|c|c|c|c|}
\hline \multirow{2}{*}{ Dimensiones } & \multicolumn{2}{|c|}{ Muestra total } & \multicolumn{2}{|c|}{ Varones } & \multicolumn{2}{|c|}{ Mujeres } \\
\hline & $M$ & $D E$ & $M$ & $D E$ & $M$ & $D E$ \\
\hline Distinción de las emociones & 2.27 & .42 & 2.31 & .41 & 2.23 & .43 \\
\hline Intercambio verbal de las emociones & 2.09 & .55 & 2.10 & .53 & 2.07 & 57 \\
\hline No ocultamiento de las emociones & 2.09 & .46 & 2.05 & .45 & 2.14 & .47 \\
\hline Conciencia corporal & 2.11 & .51 & 1.98 & .50 & 2.26 & .49 \\
\hline Atención a las emociones de los otros & 2.65 & .37 & 2.52 & .40 & 2.78 & 2.27 \\
\hline Análisis de las emociones & 2.30 & .41 & 2.26 & .43 & 2.33 & .39 \\
\hline
\end{tabular}

TABLA 2

COMPARACIÓN DEL MODELO A TRAVÉS DEL SEXO (VARONES = 687; MUJERES $=629$ )

\begin{tabular}{|l|cccccc|cccccc|}
\hline & $\chi^{2}$ & $g l$ & $p$ & $\chi^{2} / g l$ & $\Delta \chi^{2}$ & $p$ & CFI & GFI & AGFI & RMR & RMSEA & $\Delta$ CFI \\
\hline $\begin{array}{l}\text { Conciencia } \\
\text { Emocional }\end{array}$ & & & & & & & & & & & \\
$\begin{array}{l}\text { No restringido } \\
\begin{array}{l}\text { Pesos de } \\
\text { medida } \\
\text { invariantes }\end{array}\end{array}$ & 1665.55 & 780 & .000 & 2.14 & & .88 & .92 & .91 & .02 & .03 \\
\hline
\end{tabular}


TABLA 3

CARGAS FACTORIALES DE LOS DOS GRUPOS

\begin{tabular}{|c|c|c|}
\hline \multirow[t]{2}{*}{ Ítems } & \multicolumn{2}{|c|}{ Invariancia por sexo } \\
\hline & Varones & Mujeres \\
\hline \multicolumn{3}{|l|}{ Distinción de las emociones } \\
\hline Ítem 1 & .39 & .44 \\
\hline Ítem 7 & .48 & .51 \\
\hline Ítem 13 & .57 & .60 \\
\hline Ítem 19 & .60 & .62 \\
\hline Ítem 24 & .50 & .51 \\
\hline Ítem 29 & .50 & .53 \\
\hline Ítem 30 & .35 & .36 \\
\hline \multicolumn{3}{|c|}{ Intercambio verbal de las emociones } \\
\hline Ítem 2 & .58 & .65 \\
\hline Ítem 8 & .69 & .77 \\
\hline Ítem 14 & .58 & .69 \\
\hline \multicolumn{3}{|c|}{ No ocultamiento de las emociones } \\
\hline Ítem 3 & .43 & .44 \\
\hline Ítem 9 & .67 & .71 \\
\hline Ítem 15 & .65 & .73 \\
\hline Ítem 20 & .61 & .66 \\
\hline Ítem 25 & .48 & .53 \\
\hline \multicolumn{3}{|l|}{ Conciencia corporal } \\
\hline Ítem 4 & .46 & .50 \\
\hline Ítem 10 & .65 & .71 \\
\hline Ítem 16 & .32 & .41 \\
\hline Ítem 21 & .68 & .74 \\
\hline Ítem 26 & .54 & .58 \\
\hline \multicolumn{3}{|c|}{$\begin{array}{l}\text { Atención a las emociones de los otros } \\
\text { Ítem } 5\end{array}$} \\
\hline Ítem 11 & .67 & .58 \\
\hline Ítem 17 & .55 & .56 \\
\hline Ítem 22 & .65 & .55 \\
\hline \multirow[t]{2}{*}{ Ítem 27} & .54 & .46 \\
\hline & .47 & .32 \\
\hline \multicolumn{3}{|l|}{ Análisis de las emociones } \\
\hline Ítem 6 & .56 & .55 \\
\hline Ítem 12 & .45 & .45 \\
\hline Ítem 18 & .56 & .55 \\
\hline Ítem 23 & .53 & .56 \\
\hline Ítem 28 & .59 & 61 \\
\hline
\end{tabular}


Samper-García, Mesurado, Richaud y Llorca

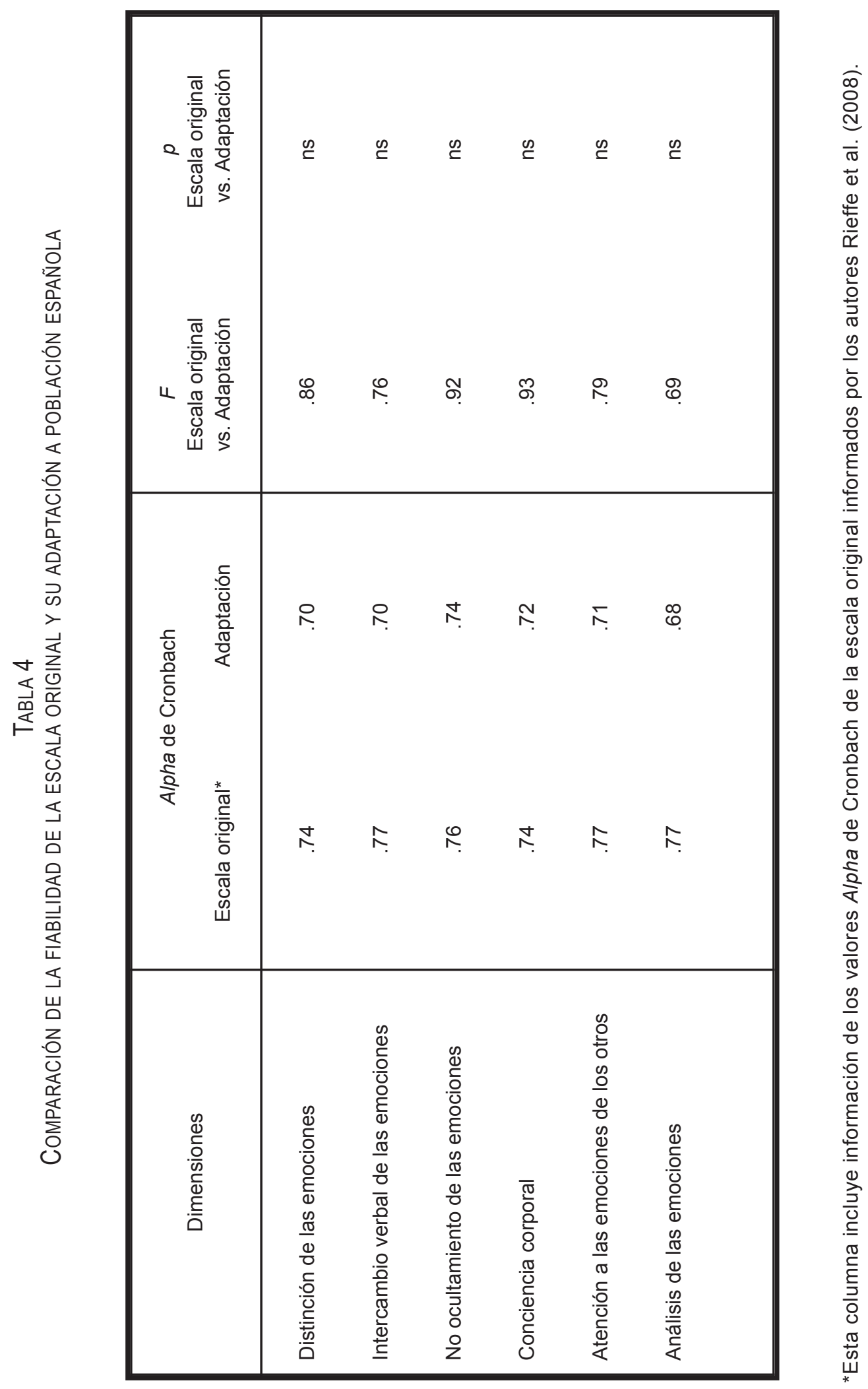




\section{ANEXO 1}

\section{Protocolo del Cuestionario de Conciencia Emocional}

A continuación, encontrarás algunas frases cortas. Cada frase es una afirmación sobre cómo puedes sentir o pensar acerca de tus sentimientos. Puedes marcar en cada frase si es verdad, algunas veces verdad o falso en tu caso. Elige la respuesta que mejor te describa. Sólo puedes marcar una respuesta. Si te resulta difícil, elige la respuesta que mejor te describa la mayoría de las veces. Por lo tanto, no hay respuestas buenas o malas, porque se trata simplemente de lo que tú piensas.

\begin{tabular}{|c|c|c|c|}
\hline Ítem & Falso & $\begin{array}{c}\text { A veces es } \\
\text { verdad }\end{array}$ & Verdad \\
\hline $\begin{array}{l}\text { 1.- A menudo estoy confundido/a o extrañado/a por lo que estoy sin- } \\
\text { tiendo. * } \\
\text { 2.- Encuentro difícil explicarle a un/a amigo/a cómo me siento. * } \\
\text { 3.- Las demás personas no necesitan saber cómo me siento. * } \\
\text { 4.- Cuando estoy asustado/a o nervioso/a, siento algo en la barriga. } \\
\text { 5.- Es importante saber cómo se sienten mis amigos. } \\
\text { 6.- Cuando estoy enfadado/a o disgustado/a, intento entender por qué. } \\
\text { 7.- Es difícil saber si me siento triste, enfadado o algo más. * } \\
\text { 8.- Me cuesta hablar con alguien de cómo me siento. * } \\
\text { 9.- Cuando estoy disgustado/a por algo, a menudo me lo guardo para } \\
\text { mí mismo/a. * } \\
\text { 10.- Cuando estoy disgustado/a, también puedo notarlo en mi cuerpo. } \\
\text { 11.- No quiero saber cómo se sienten mis amigos/as. * } \\
\text { 12.- Mis sentimientos me ayudan a entender qué ha pasado. } \\
\text { 13.-Nunca sé exactamente qué clase de sentimiento estoy experimen- } \\
\text { tando.* } \\
\text { 14.- Puedo explicar fácilmente a un/a amigo/a cómo me siento por den- } \\
\text { tro. } \\
\text { 15.- Cuando estoy enfadado/a o disgustado/a, intento esconderlo. * } \\
\text { 16.- No siento nada en mi cuerpo cuando estoy asustado/a o nervioso/a. * } \\
\text { 17.- Si un/a amigo/a está disgustado/a, intento entender por qué. } \\
\text { 18.- Cuando tengo un problema, me ayuda saber cómo me siento ante } \\
\text { el problema. } \\
\text { 19.- Cuando estoy disgustado/a, no sé si estoy triste, asustado/a o enfa- } \\
\text { dado/a. * } \\
\text { 20.- Cuando estoy disgustado/a, intento no mostrarlo. * }\end{array}$ & & & \\
\hline
\end{tabular}

Continúa 


\begin{tabular}{|c|c|c|c|}
\hline İtem & Falso & $\begin{array}{c}\text { A veces es } \\
\text { verdad }\end{array}$ & Verdad \\
\hline $\begin{array}{l}\text { 21.- Noto mi cuerpo diferente cuando estoy disgustado/a con algo. } \\
\text { 22.- No me importa cómo se sienten mis amigos/as por dentro. * } \\
\text { 23.- Es importante conocer cómo me siento. } \\
\text { 24.- A veces estoy disgustado/a, y no tengo ni idea de por qué. * } \\
\text { 25.- Cuando me siento mal, no es asunto de nadie más. * } \\
\text { 26.- Cuando estoy triste, noto mi cuerpo débil. } \\
\text { 27.- Por lo general, sé cómo se sienten mis amigos/as. } \\
\text { 28.- Siempre quiero saber por qué me siento mal con algo. } \\
\text { 29.- A menudo no sé por qué estoy enfadado/a. * } \\
\text { 30.- No sé cuándo algo me va a disgustar o no. * }\end{array}$ & & & \\
\hline
\end{tabular}

\section{Nota:}

* Ítems inversos.

Distinción de las emociones: ítems 1, 7, 13, 19, 24, $29,30$.

Intercambio verbal de las emociones: ítems 2, 8, 14.

No ocultamiento de las emociones: ítems 3, 9, 15, 20, 25.

Conciencia corporal: ítems 4, 10, 16, 21, 26.

Análisis de las emociones: ítems 6, 12, 18, 23, 28.

Atención a las emociones de los otros: ítems 5, 11, 17, 22, 27. 


\section{REFERENCIAS BIBLIOGRÁFICAS}

Arbuckle, J.L. (2006). Amos 7.0 user's guide. Chicago: SPSS.

Cole, P.M., Michel, M.K. \& Teti, L.O. (1994). The development of emotion regulation and dysregulation: A clinical perspective. Monographs of the Society for Research in Child Development, 59(2-3), 73-102. http://dx.doi. org/10.1111/j.1540-5834.1994.tb01278.x

Eisenberg, N., Hofer, C. \& Vaughan, J. (2007). Effortful control and its socioemotional consequences. En J. Gross (Ed.), Handbook of emotion regulation (pp. 287-306). New York, NY: Guilford.

Fabes, R.A., Eisenberg, N., Jones, S., Smith, M., Guthrie, I., Poulin, R., Shepard, S. \& Friedman, J. (1999). Regulation, emotionality, and preschoolers' socially competent peer interactions. Child Development, 70, 432-442.

Ferragut, M. \& Fierro, A. (2009). Inteligencia emocional, bienestar personal y rendimiento académico en preadolescentes [Emotional intelligence, personal well-being and academic performance in preadolescents]. Revista Latinoamericana de Psicología, 44(3), 95104.

Hambleton, R.K. (2001). The next generation of the ITC Test translation and adaptation guidelines. European Journal of Psychological Assessment, 17, 164-172. http://dx.doi.org/10. 1027//1015-5759.17.3.164

Henry, J.L. \& Crawford, J.R. (2005). The shortform version of the Depression Anxiety Stress Scales (DASS-21): Construct validity and normative data in a large non-clinical sample. British Journal of Clinical Psychology, 44, 277-239. http://dx.doi.org/10.1348/0144665 $05 \times 29657$

Hu, L. \& Bentler, P.M. (1999). Cutoff criteria for fit indexes in covariance structure analysis: Conventional criteria versus new alternatives. Structural Equation Modeling, 6, 1-55. http:// dx.doi.org/10.1080/10705519909540118

Jiménez Morales, M.I. \& López-Zafra, E. (2007). Inteligencia emocional y rendimiento escolar:
Estado actual de la cuestión [Emotional intelligence and school performance: Current status of the issue]. Revista Latinoamericana de Psicología, 41(1), 67-77.

Kline, R.B. (1998). Principles and practice of structural equation modeling. New York: The Guilford Press.

Koole, S.L. (2009). The psychology of emotion regulation: An integrative review. Cognition and Emotion, 1, 4-41. http://dx.doi.org/10.1 080/02699930802619031

Lahaye, M., Luminet, O., Broeck, N.V., Bodart, E. \& Mikolajczak, M. (2010). Psychometric properties of the Emotion Awareness Questionnaire for Children in a French-Speaking Population. Journal of Personality Assessment, 92(4), 317-326. http://dx.doi.org/10.1 080/00223891.2010.482003.

Lahaye, M., Mikolajczak, M., Rieffe, C., Villanueva, L., Broeck, N.V., Bodart, E. et al. (2011). Cross-validation of the Emotion Awareness Questionnaire for Children in Three Populations. Journal of Psychoeducational Assessment, 29(5), 418-427. http://dx.doi.org/10.1 177/0734282910390013

Mikolajczak, M., Roy, E., Luminet, O., Fillee, C. $\&$ de Timary, P. (2007). The moderating impact of emotional intelligence on free cortisol responses tostress. Psychoneuroendocrinology, 32(8-10), 1000-1012.

Milfont, T.L. \& Fischer, R. (2010). Testing measurment invariance across groups: Applications in cross-cultural research. International Journal of Psychological Research, 3(1), 111121.

Rieffe, C., Oosterveld, P. \& Meerum Terwogt, M. (2006). An alexithymia questionnaire for children: Factorial and concurrent validation results. Personality and Individual Differences, 40, 123-133. http://dx.doi.org/10.1016/j.paid.20 05.05.013

Rieffe, C., Oosterveld, P., Miers, A.C., Terwogt, M.M. \& Ly, V. (2008). Emotion awareness and internalising symptoms in children and adolescents: The Emotion Awareness Questionnaire revised. Personality and Individual Differ- 
ences, 45, 756-761. http://dx.doi.org/10.1 016/ j.paid. 2008.08.001

Rieffe, C., Terwogt, M.M., Petrides, K.V., Cowan, R., Miers, A.C. \& Tolland, A. (2007). Psychometric properties of the Emotion Awareness Questionnaire for children. Personality and Individual Differences, 43, 95-105. http://dx. doi.org/10.1080/00223891.2010.482003.

Rowley, A.A., Roesch, S.C., Jurica, B.J. \& Vaughn, A.A. (2005). Developing and validating a stress appraisal measure for minority adolescents. Journal of Adolescence, 28, 547557. http://dx.doi.org/10.1016/j.adolescence. 2004.10.010

Rueda, B., Pérez-García, A.M., Sanjuan, P. \& Ruiz, M.A. (2006). Relación entre la alexitimia y hostilidad: ¿Una asociación influida por el malestar psicológico? [Relationship between alexithymia and hostility: An association influenced by psychological distress?]. Análisis y Modificación de la Conducta, 32 (143), 264-286.

Salovey, P., Stroud, L.R.,Woolery, A. \& Epel, E.S. (2002). Perceived emotional intelligence, stress reactivity, and symptom reports: Further explorations using the trait meta-mood scale.
Psychology \& Health, 17, 611-627. http://dx. doi.org/10.1080/08870440290025812

Schutte, N.S., Malouff, J.M., Thorsteinsson, E.B., Bhullar, N. \& Rooke, S.E. (2007). A meta-analytic investigation of the relationship between emotional intelligence and health. Personality and Individual Differences, 42, 921-933. http:// dx.doi.org/10.1016/j.paid.20 06.09.003

Sifneos, P.E. (1973). The prevalence of alexithymic characteristics in psychosomatic patients. Psychotherapy and Psychosomatics, 22, 255262. http://dx.doi.org/10.1159/000286529

Thompson, R.A. \& Meyer, S. (2007). Socialization of emotion regulation in the family. En J. Gross (Ed.), Handbook of emotion regulation (pp. 249-268). New York, NY: Guilford. van der Vijver, F. \& Leung, K. (1997): Methods and Data Analysis for Cross-Cultural Research. London: SAGE Publications.

Zeman, J., Cassano, M., Perry-Parrish, C. \& Stegall, S. (2006). Emotion regulation in children and adolescents. Journal of Developmental and Behavioral Pediatrics, 27, 155168. http://dx.doi.org/10.1097/00004703-200 604000-00014

Universitat de Valencia Valencia - España

Fecha de recepción: 7 de octubre de 2015 Fecha de aceptación: 10 de febrero de 2016 